\title{
SCANNING STRATEGIES IN THE PBF PROCESS - A CRITICAL REVIEW
}

\author{
Ehsan Malekipour ${ }^{1}$ \\ School of Mechanical Engineering, Purdue University \\ West Lafayette, IN 47907, USA
}

\author{
Hazim El-Mounayri \\ Purdue School of Engineering and Technology \\ Indianapolis, IN 46202, USA
}

\begin{abstract}
The powder-bed fusion (PBF) process is capable of producing near-fully dense metallic parts; however, various defects -- particularly thermal abnormalities -- can still be observed during the process. Some of these thermal defects -cracks, distortion, delamination of layers, and microporosity -cannot be removed by post-processing operations. The majority of these abnormalities are the result of residual stress, heat accumulation, lack of inter-track /inter-layer bonding, lack of powder fusion, or a combination of these factors. Modifying the scanning strategy (the topology of scanning tracks) can efficiently mitigate these abnormalities by adjusting the process parameters and adopting proper scanning patterns.

The implementation of different scanning strategies significantly changes the ultimate quality of printed parts and manufacturing process lead time. Choosing a proper scanning strategy minimizes the residual stress and internal porosity, generates homogeneous microstructure, and avoids heat accumulation throughout the part during the printing process.

In this work, we conducted a critical review of different scanning strategies, their pros and cons, limitations, and influence on the resulting properties of fabricated parts. Furthermore, we report the latest efforts for improvement of the current scanning strategies and introduce the-state-of-the-art strategies in the multi-laser PBF (ML-PBF) process. The insights provided here can assist scholars in evaluating existing scanning strategies and scanning patterns, and in identifying ways both to overcome scanning limitations and to modify them. On the other hand, it can assist manufacturers in selecting the best scanning strategies for their products based on their designs, demands, and resources.
\end{abstract}

Keywords: powder-bed fusion; metal additive manufacturing; scanning strategy; scanning pattern; multi-laser and single-laser AM; residual stress; temperature distribution

\section{INTRODUCTION}

A vast number of industries have been employing the powder-bed fusion (PBF) process. However, their abilities to improve the lead time were significantly limited due to thermal abnormalities generated during the process [1]. The majority of these abnormalities are caused by the prevalence of non-uniform temperature distribution [2], which produces temperature gradients between different zones throughout a layer [3]. This gradient induces residual stresses in these zones, and the stress is increased as more layers are added, resulting in distortion, warping, and detachment of the part from its supports, and eventually failure (see Figure 1) [3-5].
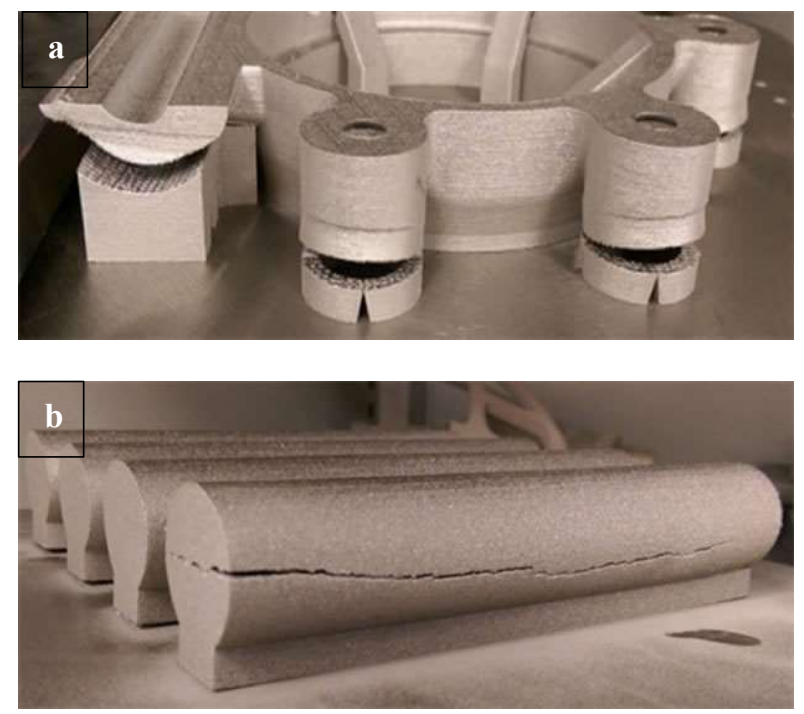

FIGURE 1: THERMAL DEFECTS DUE TO RESIDUAL STRESS [4]

${ }^{1}$ Corresponding author: emalekip@purdue.edu

This is the author's manuscript of the article published in final edited form as:

Malekipour, E., \& El-Mounayri, H. (2021, February 16). Scanning Strategies in the PBF Process: A Critical Review. ASME 2020 International Mechanical Engineering Congress and Exposition. https://doi.org/10.1115/IMECE2020-24589 
Literature shows different methods that can be implemented to mitigate the effects of residual stress on the printed part: preheating of the baseplate and/or powder bed, rescanning the printed layer, utilizing post-processing heat treatment, and manipulating the scanning strategy. However, preheating and rescanning decrease energy efficiency and increase manufacturing time and total cost $[6,7]$. Heat treatment can reduce the residual stress up to $70 \%$ as reported by Shiomi et al. [8]; however, it is incapable of reversing the effects of residual stresses on distortions, delamination, and stress-related cracking that occur during the manufacturing process [7]. Some experimental observations suggest limiting the dimensions of the printed components, modifying the part design, or adding support structures to avoid distortion and part failure. However, all of these approaches are either uncertain, increase the manufacturing cost and time, and/or are based on trial and error methodology.

One of the most attractive in situ methods to effectively mitigate the influence of residual stress is scanning strategy optimization. Scanning strategy refers to the manipulation of both laser specifications -- laser power, scanning speed, etc. -and the laser scanning pattern topology which prints different zones of the exposure layer. In addition to having a direct influence on residual stresses, scanning strategies significantly impact the ultimate quality of the fabricated part in such properties as achievable density, microstructure, and surface finish. In the following sections, we provide both a critical review of different scanning strategies and an explanation of their effect on scanning properties.

\section{PREVALENT SCANNING PATTERNS}

To print each layer, the scanning algorithms split the exposure layer into various regions based on the specific exposure area. In short, these algorithms define each area as core, skin, up-skin, or down-skin based on the existence of material above or below the layer. Consequently, the algorithms assign different process parameters to different regions such as powder zones, re-solidified material, thin wall, larger solid volume, etc. [9]. Furthermore, each algorithm assigns a scanning pattern to print each zone. Patterns are designed to minimize porosity, generate uniform temperature distribution, and avoid overheating throughout the exposure zones. Non-uniform temperature distribution or overheated zones mainly happen either in the layers with complex geometry in the parts with long dimensions, on the overhangs which lack support structure due to lack of heat sink, or on longer scanning tracks over which the shear forces can act [3, 4, 10]. Numerous literature reference highlights the influence of different scanning patterns on the thermal distribution of an exposure layer. They revealed that adopting the proper scanning pattern mitigates the generated residual stresses and thermal distortion since various patterns affect the length of the scanning vectors and, hence, the level of stress generated in the component. In general, there are five prevalent scanning patterns: simple rastering, bi-directional, offset-out, offset-in, fractal, (see Figure 2.a to Figure 2.e) [5]. Each pattern can scan an entire layer or the separated islands or stripe in the chessboard or stripe scanning pattern (see Figure 2.f and Figure 2.g) $[5,11,12]$.

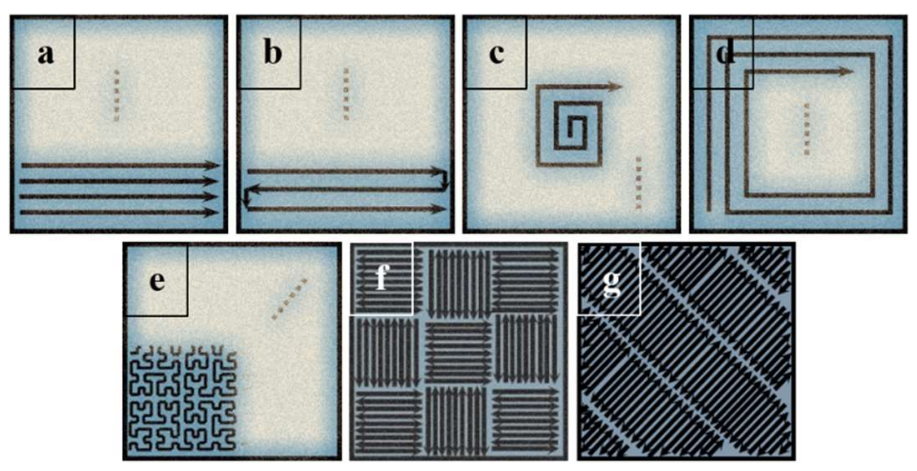

FIGURE 2: DIFFERENT SCANNING PATTERNS

\section{SCANNING STRATEGIES AND THEIR OVERALL EFFECTS}

\subsection{Residual stress for different patterns}

Parry et al. [13] in 2016 demonstrated that the longitudinal stress (parallel to the scanning vector) is the main contributor to residual stress due to both the larger thermal gradient and the scanning vector which increases with the vector length. They did not observe a substantial difference in the magnitude of stress caused by different scanning strategies in the PBF process; however, they showed a non-uniform anisotropic stress field which was caused by the differing thermal history in different scanning strategies. Cheng et al. [14] in 2016 studied the effects of different scanning patterns on residual stress and deformation of a printed layer in the SLM process. Their simulation results demonstrated that the parts printed by $45^{\circ}$ inclined lines possess the minimum residual stresses in both $\mathrm{X}$ and $\mathrm{Y}$ directions (Figure 3.a) as well as the lowest deflection (Figure 3.b). However, they scanned the islands with a $90^{\circ}$ instead of a $45^{\circ}$ orientation which is the suggested printing orientation in previous literature. Parry et al. [13] via their thermo-mechanical simulation demonstrated anisotropic residual stress in parts printed by the PBF process due to nonuniform thermal history. This stress typically increases with longer tracks. Cheng et al. [14] and Nickel et al. [15], on the other hand, numerically demonstrated that the employment of the chessboard scanning strategy with rotating scan patterns can result in a more homogeneous residual stress distribution.

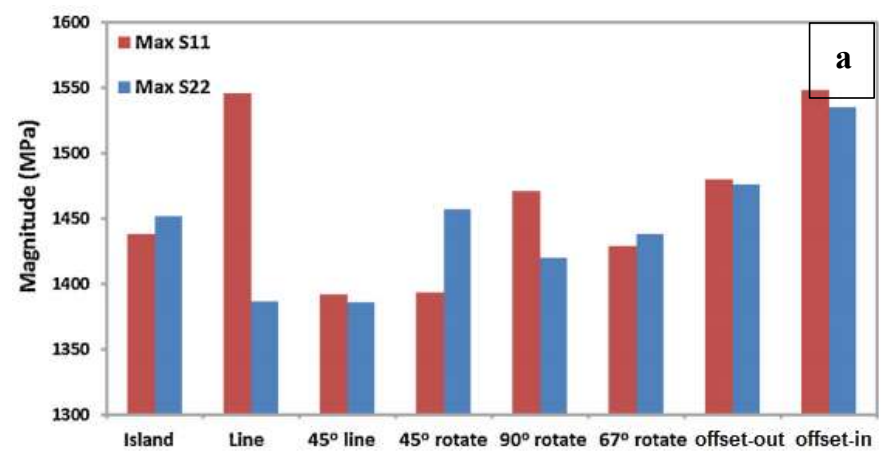




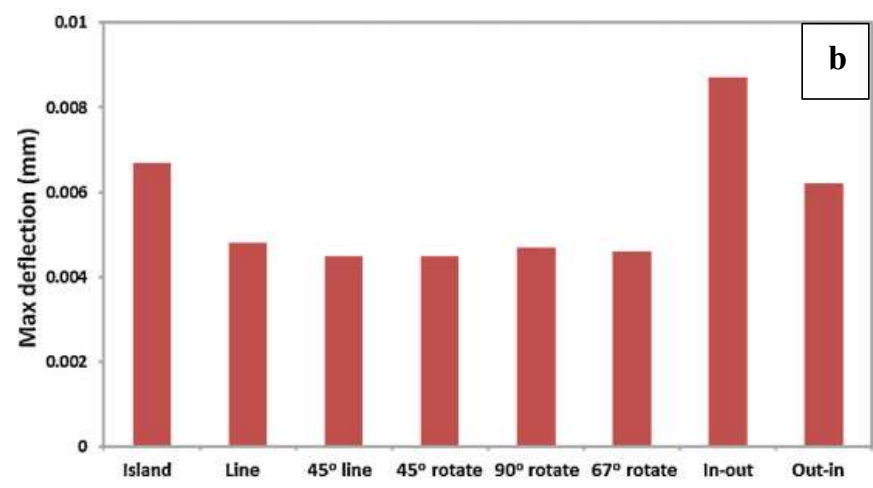

FIGURE 3: A. RESIDUAL STRESS IN X (RED) AND Y (BLUE) DIRECTIONS AND B. MAXIMUM DEFLECTION FOR DIFFERENT SCANNING STRATEGIES [14]

\subsection{Chessboard strategy versus stripe scanning strategy}

The chessboard scanning pattern segments an exposurelayer into identical imaginary square sections called islands. The default strategies in the current $3 \mathrm{D}$ printers scan each island by a simple rastering or bi-directional scanning pattern with vectors that are perpendicular to the vectors in the neighboring islands. The stripe scanning pattern, on the other hand, is a band in which the scanning vector width is the same as the stripe width with overlap between the neighboring stripes. Stripe and chessboard scanning keep the lengths of individual scan lines shorter, generate homogenous residual stress which reduces the gradual build-up of residual stress and is appropriate for parts with large dimensions. In general, the chessboard scanning strategy generates less residual stress than stripe strategy since it successfully reduces the localized thermal gradients and, subsequently, the generated residual stress $[16,17]$. However, the stripe scanning strategy has a higher manufacturing-rate compared with the island strategy and increases the part density $[4,14,18]$. The scanning strategies usually have translational shift and rotate the orientation of the scanning vectors $67^{\circ}$ from one layer to another so that stresses are not all aligned in the same plane; thus, it is many layers before the scanning direction is exactly repeated [4].

There are four typical scanning sequences of the islands in the chessboard scanning strategy introduced by the previous literature, namely, default Island Strategy (introduced by Concept Laser M2 Scanning Strategy), Successive Scanning Strategy, Successive Chessboard Scanning Strategy, and Lease Heat Influence (LHI) Chessboard Scanning Strategy (see Figure 4) [7]. The first strategy scans the islands in a random order [19](Figure 4.a). The second strategy scans the islands consecutively (Figure 4.b). The light and dark islands represent the $90^{\circ}$ rotation of scanning vectors in adjacent islands [20,21]. The third scanning strategy scans the islands in a chessboard fashion [22] (Figure 4.c). The fourth strategy is a variation of the successive chessboard strategy in which the next island to be printed lies as far as possible from the current island $[18,20]$ (Figure 4.d).

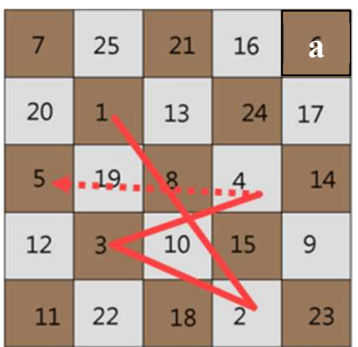

a. Island (approximation)

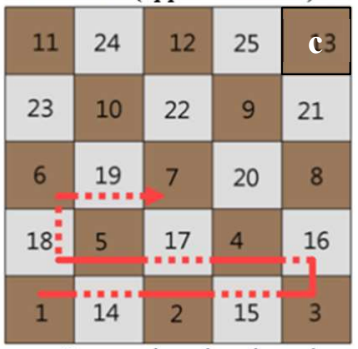

c. Successive chessboard

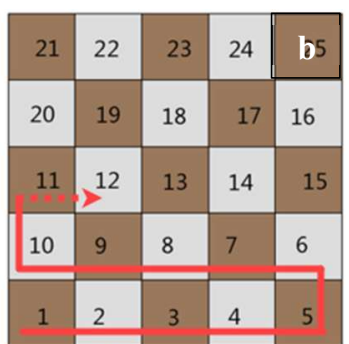

b. Successive

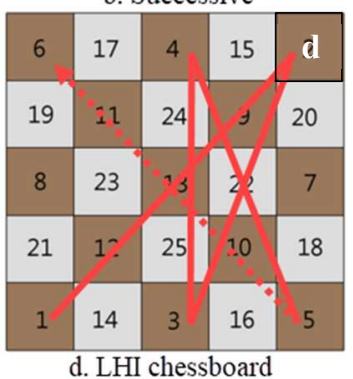

FIGURE 4: TYPICAL SCANNING SEQUENCES IN THE CHESSBOARD SCANNING STRATEGY [7]

Some scholars strive to improve the chessboard scanning strategy by trying different printing orders or scanning patterns for the islands. Hagedorn-Hansen et al. [23] in 2017 developed an island scanning strategy called "varying-helix island scanning strategy" and experimentally compared its warpage and porosity with the default island scanning strategy. This strategy begins scanning of all the islands placed in set 1 and continues printing the remaining sets in the order shown in Figure 5.a to finish all the islands. The results showed that the new strategy could reduce the deviation around $30 \%$ and the porosity decreased from $0.740 \%$ to $0.413 \%$. However, this strategy increased manufacturing time significantly. In another effort, Valente et al. [24] tried a chessboard scanning strategy with a specific six different random scanning patterns for the islands called the modeled island scanning strategy (Figure 5.b). This strategy reduced the porosity compared with the island scanning strategy by a significant amount due to the lack of fusion.

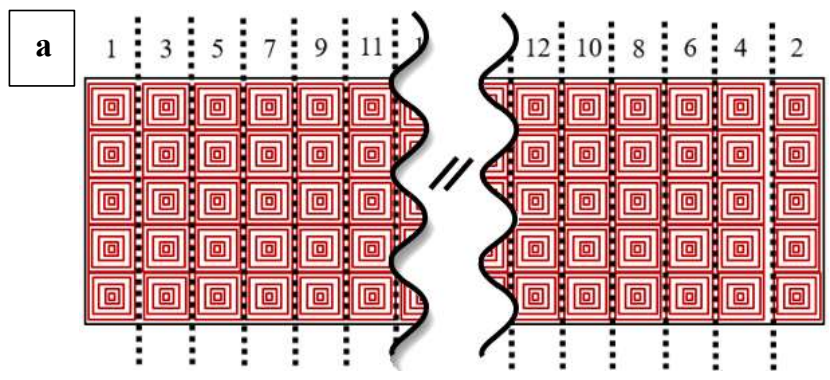




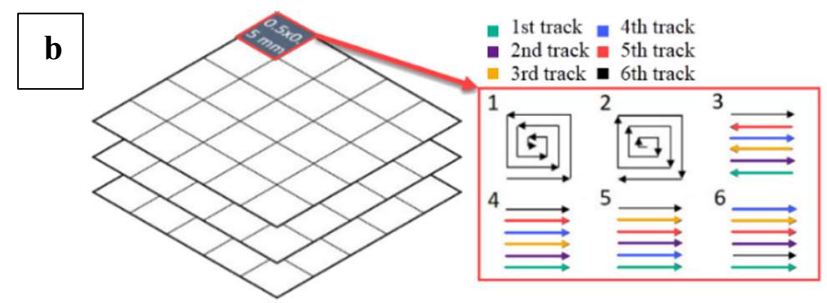

FIGURE 5: A. VARYING-HELIX ISLAND SCANNING STRATEGY [23]; B. THE MODELED ISLAND SCANNING STRATEGY [24]

Mugwagwa et al. [7] in 2019 used the x-ray diffraction technique to measure the residual stress in all the typical scanning strategies. As shown in Figure 6, the successive chessboard scanning strategy caused the fewest average residual stresses and lowered residual stress by up to $40 \%$ compared with the default island strategy. Figure 6 also shows that the residual stress distribution in the PBF process is highly anisotropic; therefore, rotating scanning vectors between adjacent islands significantly helps to minimize the anisotropic residual stress. Moreover, they demonstrated that the successive chessboard strategy leads to lower form deviations compared with the island strategy.

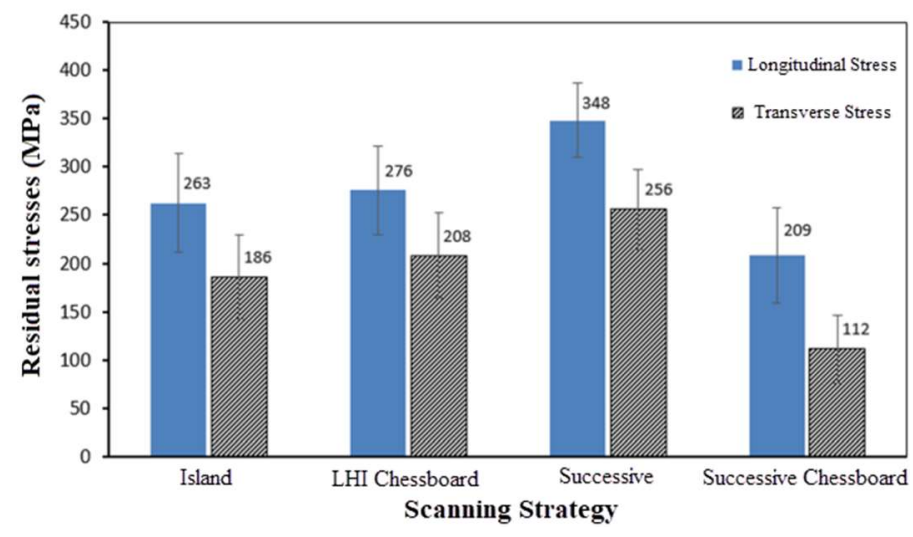

FIGURE 6: COMPARISON OF RESIDUAL STRESS FOR DIFFERENT CHESSBOARD SCANNING STRATEGIES [7]

\subsection{Island factors and their effects on part quality}

Yasa et al. [11] in 2012 studied the effects of island orientation on the residual stress in the chessboard scanning pattern. The results showed that the orientation of the islands significantly affects the resulting residual stress. Their experiments demonstrated that a $45^{\circ}$ rotation in islands relative to the $\mathrm{x}$-axis reduces the thermal stress by $36 \%$ compared with the reference part scanned by long scanning vectors. In this work, the laser scanned the islands in random order. In a 2015 study, Lu et al. [25] investigated the effects of islands with the sizes of $2 \times 2,3 \times 3,5 \times 5$, and $7 \times 7 \mathrm{~mm}^{2}$ on the microstructure, mechanical properties, and residual stress of a part printed by the SLM process. Their results suggested that $5 \times 5 \mathrm{~mm}^{2}$ is the optimal size for the islands in the chessboard scanning strategy.
This size is the default size in the current $3 \mathrm{D}$ printing machines. Figure 7 shows the optical morphologies of the islands with different sizes in a part printed by the SLM process. In late 2017, Ali et al. [22] studied the effects of different sizes of islands (scanning vector length) and scanning vector rotation. Their results showed that an increase in the scanning vector length increases the residual stress while rotation of the vectors in adjacent islands and successive layers reduces the residual stress. The scanning strategy with perpendicular scanning vectors in adjacent islands and $90^{\circ}$ rotation in successive layers resulted in the lowest residual stress.

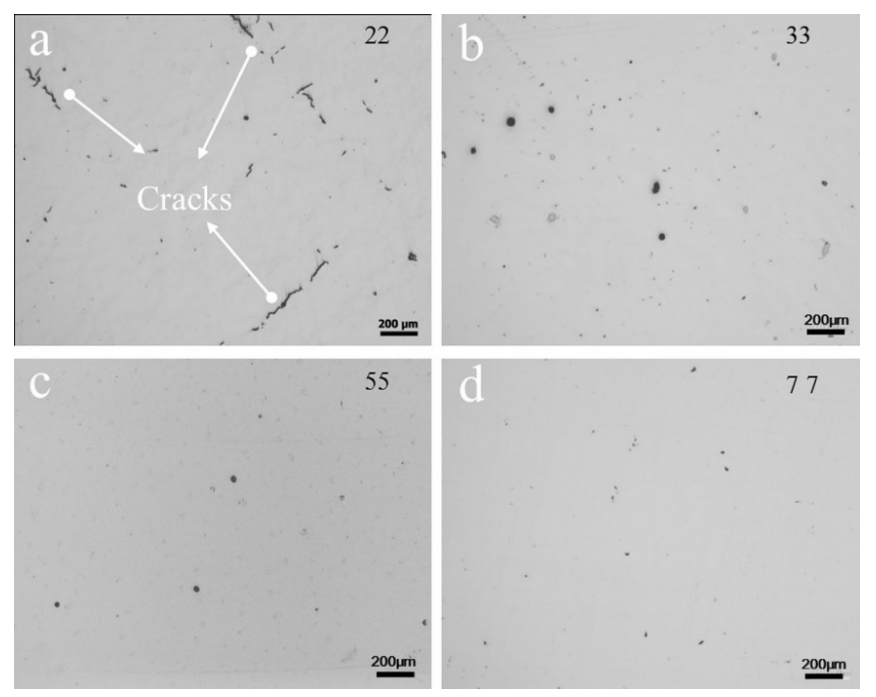

FIGURE 7: THE OPTICAL MORPHOLOGIES OF THE SELECTIVE LASER MELTED INCONEL-718 SPECIMENS: (A) 22SPECIMEN; (b) 33-SPECIMEN; (c) 55-SPECIMEN; (d) 77SPECIMEN [2]

\subsection{Scanning strategies and manufacturing time}

Mugwagwa et al. [7] evaluated the impact of different scanning strategies on the scanning times required for printing a $50 \times 50 \mathrm{~mm}$ scanning area. Although the differences for the various strategies are only fractions of a second, it will increase proportionally for parts with larger dimensions or complex geometry. Their evaluation showed that successive strategy is the fastest strategy. However, successive chessboard strategy is the most promising chessboard scanning strategy since it generates the least residual stress (see section 3.2) while the scanning time is lower than either the island or LHI chessboard strategies.

TABLE 1: SCANNING TIMES RELATED WITH DIFFERENT SCANNING STRATEGIES [7]

\begin{tabular}{ll}
\hline Scanning strategy & Scanning time (sec) \\
\hline Island strategy & 40.54 \\
\hline Successive strategy & 40.04 \\
\hline Successive chessboard strategy & 40.16 \\
\hline LHI chessboard strategy & 40.68 \\
\hline
\end{tabular}




\subsection{Scanning strategies and microstructure}

Valente et al. [24] in 2019 employed three different scanning strategies (bi-directional, island strategy, and modeled island strategies) and demonstrated that different scanning strategies affect the surface texture, porosity, and microstructure of the manufactured part. Their experiments showed that amongst the three scanning strategies, the bi-directional scanning strategy generated the lowest surface roughness but distributed unevenly; while the roughness increased with the use of island strategy, the texture was more evenly distributed (Figure 8).

Similarly, as Figure 8 shows, a low amount of porosity was found in the specimen printed by the bi-directional strategy $(0.4 \%)$, mainly consisting of spherical pores, while $1.4 \%$ and $0.8 \%$ were found in the specimen printed by the island strategy

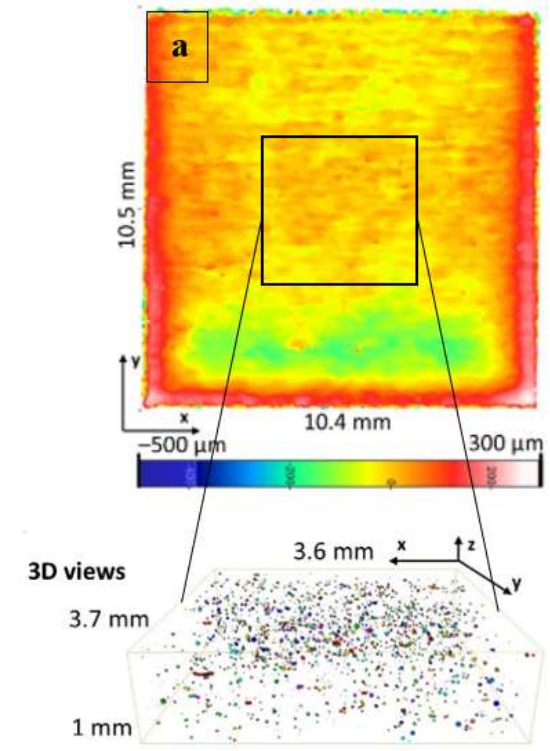

and modeled island strategy, respectively. Lack of fusion was the main source of porosity in the island strategy while it was reduced significantly by employment of the modeled strategy which mainly created spherical pores as the main porosity type. They also reported that scan strategies affected the thermal histories of the specimens and determined the microstructure. This is true in all inherently thermal additive manufacturing processes [26]; however, due to a large number of porosities in the specimens printed by the island scanning strategies, it was unclear whether the difference in the thermal history is entirely caused by the difference in the employed scanning strategy or is impacted by geometry change which can cause the large porosities.

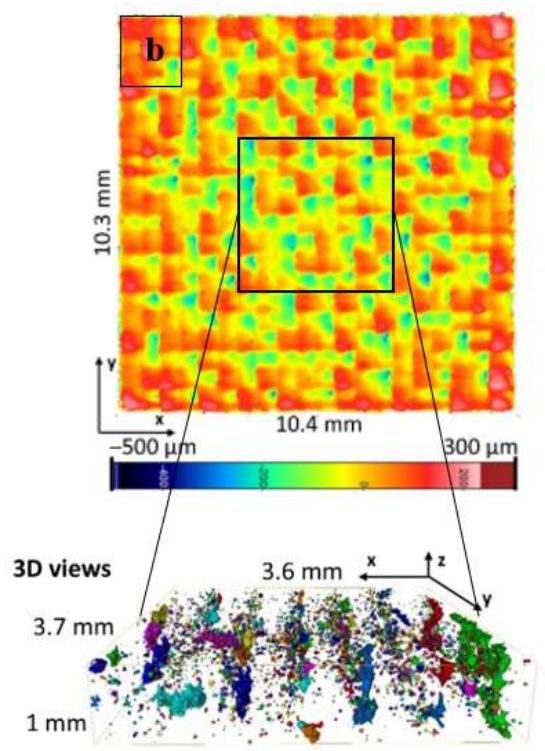

FIGURE 8: INFINITE FOCUS MICROSCOPE (IFM) IMAGE AND COMPUTED TOMOGRAPHY (CT) RESULTS FOR A. BI-DIRECTIONAL STRATEGY; B. ISLAND STRATEGY [24]

Furthermore, the previous literature demonstrated that the samples featured columnar grains independent of scanning pattern or laser power, as well as the grain size, as long as the laser power is kept constant. However, the grain size decreases as the selected laser power decreases (see Figure 8) [27].
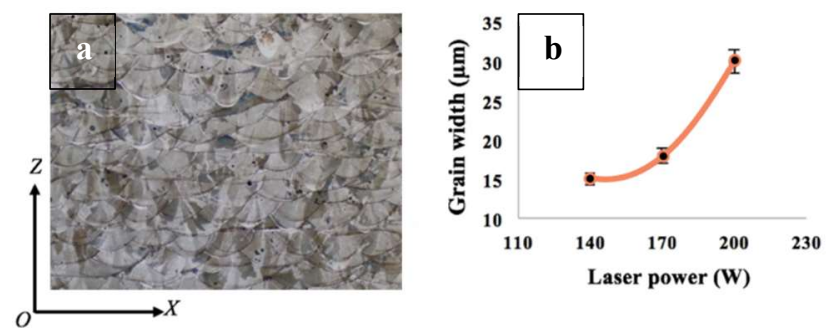

FIGURE 9: A. THE GRAIN STRUCTURE OF 316L; B. CORRELATION OF GRAIN WIDTH WITH LASER POWER [27]

\section{Continuous laser scanning strategies}

The National Institute of Standards and Technology (NIST) developed an open architecture Additive Manufacturing Metrology Testbed (AMMT) which provides an open architecture for flexible monitoring and control during the PBF process. AMMT allows scholars to control scanning strategies, including laser power and scanning speed, within each scan line. Yeung et al. in 2017 [28] leveraged the testbed to introduce a 'continuous' scanning strategy to reduce build times by removing the need to turn the laser off between scan tracks. In continuous strategy, two sequential moves with different velocities are connected by an arc to smoothly transient the velocity between the move. This strategy is the opposite of the default strategy, constant build speed move or sky-writing. In the latter strategy, the velocity is kept constant for the entire motion while the laser is on; thus, the strategy may need to add extra moves before or after the original move to increase or decrease the velocity while it turns the laser off. They introduced and 
implemented continuous linear, continuous concentric, continuous linear-island, and continuous concentric-island on AMMT and compared their results with the constant build speed (sky-writing) in conventional methodology. They considered two different scanning patterns: linear and concentric (Figure 10.a). The scanning tracks can be printed either in an interleaved manner or sequentially with alternative power (Figure 10.b). For a larger scanning area, they employed the chessboard scanning strategy with three different sequences (Figure 10.c). The results showed that the continuous scanning strategy could improve the build time $23 \%$, and the continuous concentric strategy effectively reduced bumps and holes compared with the traditional island strategy.

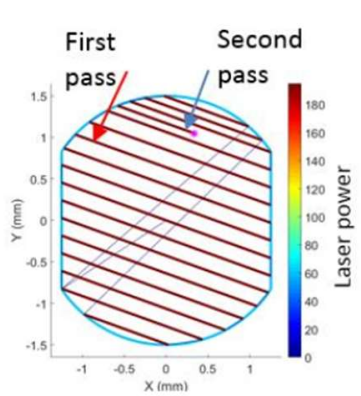

Interleaved scan

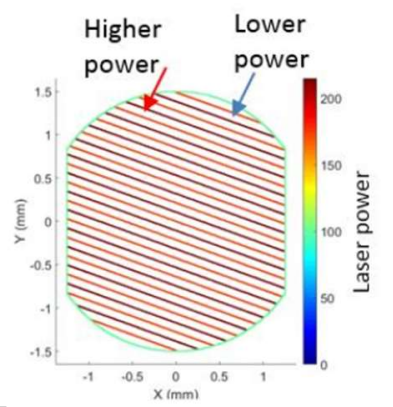

Alternative power

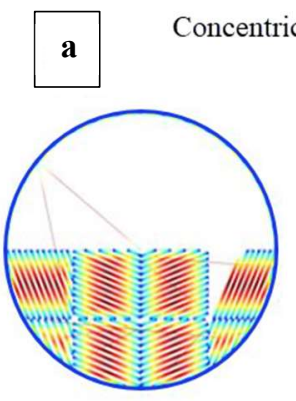

1. Sequential

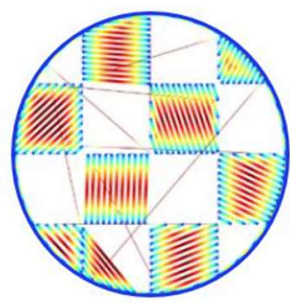

2. Chessboard

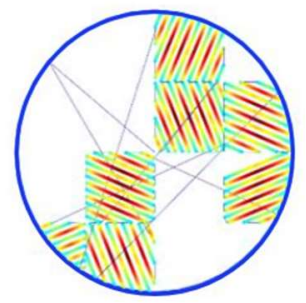

3. Random b

\author{
(1)
}

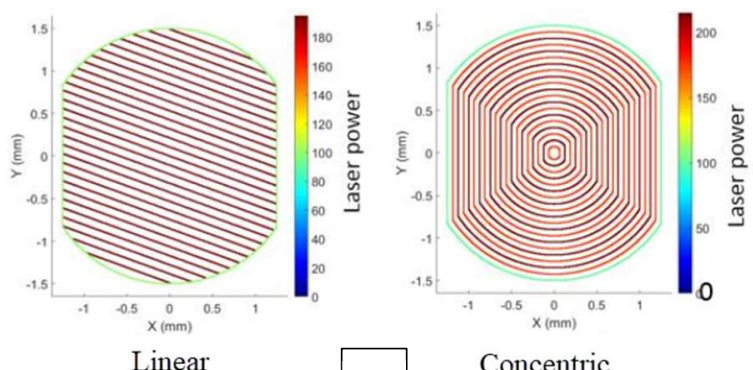

$\mathbf{a}$

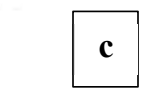

FIGURE 10: NIST AMMT CONTINUOUS SCANNING STRATEGIES: A. DIFFERENT SCANNING PATTERNS; B. DIFFERENT PATTERNS OF POWER SCAN, AND C. DIFFERENT SCANNING SEQUENCE IN CHESSBOARD STRATEGY [28]

\section{MULTI-LASER SCANNING STRATEGY}

Multi-Laser Powder Bed Fusion (ML-PBF) is a metal AM method that can significantly reduce manufacturing time. At the end of 2013, SLM Solutions presented an automated system, SLM 500HL, which was equipped with a multi-laser/multiscanner system with up to four lasers and four scanners. Using multiple lasers can reduce the time required to fabricate each layer by $75 \%$ when using four lasers [29]. One factor that can help expedite the manufacturing process is the possibility of manufacturing of support structure with a thicker layer (due to the employment of a laser with different laser power). Masoomi et al. [30] in 2017 studied the influence of various single-laser and multi-laser PBF scan strategies on heat-affected zones (HAZs), melt pool temperatures, and local temperature gradient magnitudes in an exposure layer. Preliminary results demonstrated that the melt pool peak temperature is insensitive while temperature gradient magnitudes are extremely sensitive to scan strategy and number of lasers. They demonstrated that employing a chessboard scanning strategy and multiple lasers reduce not only the average magnitude of peak temperature gradients experienced but also the general level of residual stress expected. However, they reported that such strategies can result in non-uniform, anisotropic residual stress distributions in printed parts, ultimately affecting their mechanical properties.

The radar charts in Figure 11 depict the uniformity in temperature gradients along with each layer in 16 different points. Different scanning strategies use either one laser (S1-S2, S5-S6, and S9) or four lasers, and the strategies print either the entire zone as a single entity (S1 and S5), four islands (S2-S7, S9-S11), or four adjacent stripes with a different combination of track directions ( $\mathrm{x}$ - or $\mathrm{y}$ - direction). In general, strategies conducive for a lower average of the maximum temperature gradient (AMTG) are found to possess less spatial homogeneity in AMTGs as evidenced by their higher standard deviation (SDTG). This work concluded that obtaining a uniform residual stress distribution appears to be highly dependent on island geometry and island distribution, as well as the orientation of laser scan vectors between adjacent islands. 


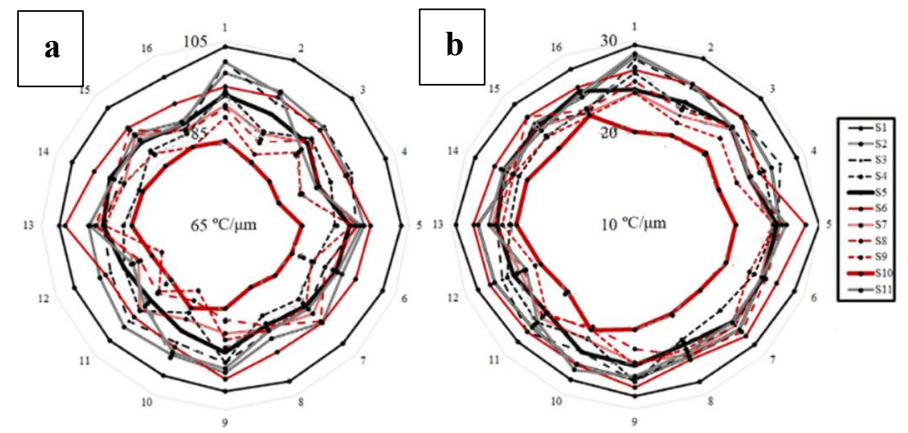

FIGURE 11: RADAR CHART SHOWING THE MAXIMUM TEMPERATURE GRADIENTS MEASURED AT POINTS P1-P16 SCANS BY THE SCAN STRATEGIES S1-S11 FOR A. THE FIRST LAYER AND B. THE SECOND LAYER [30]

\section{CURRENT LIMITATIONS ON THE COMPONENTS FINAL QUALITY}

Residual stresses and generated porosity (ultimate density) are two major concerns in the PBF process, as they can determine the warpage of artifacts after removing from the base or fail the fabricated part even at its final stage $[3,31]$. To resolve these issues, it is common practice to perform heat treatment while the part still attaches to the building platform to release the residual stresses before the removal [3,31], reduce the temperature gradients by manufacturing of proper support structure [3], and modify the scan strategies [13]. Currently, the modification of scan strategy is under the spotlight since it can increase the final quality of fabricated parts without noticeable compromising the process lead time, post-process expenses, consumed material, or cost of the manufacturing process. Table 1 shows the features and the limitations of the parts produced by the current PBF processes [31]:

\section{TABLE 2: PBF PRODUCED COMPONENTS FEATURES}

\begin{tabular}{ll}
\hline Ultimate features of fabricated parts & Current limitation \\
\hline Relative density ${ }^{2}[\%]$ & Up to 100 \\
\hline Upper surfaces roughness $(R a, X-Y)[\mu \mathrm{m}]$ & $4-10$ \\
\hline Lateral surfaces roughness $(R a, Z)[\mu \mathrm{m}]$ & $>20$ \\
\hline Minimum feature size $[\mu \mathrm{m}]$ & $75-250$ \\
\hline Geometric tolerance $[\mathrm{mm}]$ & $\pm 0.05-0.1$ \\
\hline Generated defects and contributing & $\begin{array}{l}\text { Please see the authors' } \\
\text { parameters }\end{array}$ \\
\hline Manufacturing lead time (predominantly & - Number of lasers \\
depends upon four items) & - Scanning speed \\
& - Scanning strategy \\
& - Post-processing \\
\hline
\end{tabular}

\footnotetext{
${ }^{2}$ Relative density is a ratio between the density of the material fabricated by the PBF process compared with conventional technologies (e.g., rolling, forging) [31]
}

\section{CONCLUSION AND FUTURE WORKS}

Based on the above critical review of the prevalent scanning strategies in literature, we conclude that the stripe scanning strategy with $67^{\circ}$ rotation and the successive chessboard scanning strategy with perpendicular scanning vectors in adjacent islands and $90^{\circ}$ rotation in successive layers are currently the most promising strategies. The stripe strategy achieves higher manufacturing speed and lower porosity compared to the successive chessboard scanning strategy. However, the latter results in the least residual stress and distortion in parts with large dimensions. Both of these strategies alleviate the common defects which typically occur in the PBF process by employing shorter scanning vectors and producing a more uniform temperature distribution throughout the exposurelayer.

On the other hand, the literature revealed significant gaps in the study of different aspects of the scanning strategies including but not limited to the influence of the thermal history of formerly printed layers and the geometry of exposure-layers printed by different strategies on temperature distribution of the last printed layer. These factors are crucial for control of the non-uniform anisotropic stress field generated during the process. Study of different scanning strategies for ML-PBF process [32], investigate the optimized topology of islands in chessboard scanning strategy [33], and the manipulation or design of novel scanning strategies for manufacturing of fully dense parts with higher speed are among other gaps need to be addressed by scholars.

Literature shows a promising future of the achieved benefits of modifying scanning strategy include controlling residual stress, generating homogenous microstructure, improving the surface roughness, minimizing the support structures, and reducing defects. The improvement of scanning strategy can significantly decrease both manufacturing cost and process lead time by minimizing post-processing. A decisive step toward improving scanning strategy is to develop and leverage closedloop in situ monitoring and control system for both SL-PBF and ML-PBF processes which allows real-time modification of scanning strategy considering the thermal history and evolution of the process [34]. Additional research must be conducted, both computationally and experimentally, to further clarify the correlation between the process, structure, property, and performance for various machine environments, materials, and constraints [30].

\section{REFERENCES}

[1] Malekipour, E. and H. El-Mounayri, Defects, process parameters and signatures for online monitoring and control in powder-based additive manufacturing, in Mechanics of Additive and Advanced Manufacturing, Volume 9. 2018, Springer. p. 8390. 
[2] Malekipour, E. and H. El-Mounayri, Common defects and contributing parameters in powder bed fusion AM process and their classification for online monitoring and control: a review. The International Journal of Advanced Manufacturing Technology, 2018. 95(1-4): p. 527-550.

[3] Malekipour, E., A. Tovar, and H. El-Mounayri, Heat Conduction and Geometry Topology Optimization of Support Structure in Laser-Based Additive Manufacturing, in Mechanics of Additive and Advanced Manufacturing, Volume 9. 2018, Springer. p. 17-27.

[4] Renishaw_Apply_Innovation. Design for Metal AM by Renishaw - A Beginner's Guide. 2017; Available from: http://additivemanufacturing.com/2017/08/21/design-for-metalam-by-renishaw-a-beginners-guide/.

[5] Shamsaei, N., et al., An overview of Direct Laser Deposition for additive manufacturing; Part II: Mechanical behavior, process parameter optimization and control. Additive Manufacturing, 2015. 8: p. 12-35.

[6] Papadakis, L., D. Chantzis, and K. Salonitis, On the energy efficiency of pre-heating methods in SLM/SLS processes. The International Journal of Advanced Manufacturing Technology, 2018. 95(1-4): p. 1325-1338.

[7] Mugwagwa, L., et al., Evaluation of the impact of scanning strategies on residual stresses in selective laser melting. The International Journal of Advanced Manufacturing Technology, 2019. 102(5-8): p. 2441-2450.

[8] Shiomi, M., et al., Residual stress within metallic model made by selective laser melting process. CIRP Annals, 2004. 53(1): p. 195-198.

[9] Inside_Metal_Additive_Manufacturing. Scanning patterns in $\overline{S L M}$. $201 \overline{5} ; \quad$ Available from: https://www.insidemetaladditivemanufacturing.com/blog/scanning-patterns-in-slm.

[10] Price, S., et al. Experimental temperature analysis of powder-based electron beam additive manufacturing. in 24th Annual International Solid Freeform Fabrication Symposium, Austin, TX. 2013.

[11] Yasa, E., et al. Investigation of sectoral scanning in selective laser melting. in ASME 2010 10th Biennial Conference on Engineering Systems Design and Analysis. 2010. American Society of Mechanical Engineers Digital Collection.

[12] Trevisan, F., et al., On the selective laser melting (SLM) of the AlSilOMg alloy: process, microstructure, and mechanical properties. Materials, 2017. 10(1): p. 76.

[13] Parry, L., I. Ashcroft, and R.D. Wildman, Understanding the effect of laser scan strategy on residual stress in selective laser melting through thermo-mechanical simulation. Additive Manufacturing, 2016. 12: p. 1-15.

[14] Cheng, B., S. Shrestha, and K. Chou, Stress and deformation evaluations of scanning strategy effect in selective laser melting. Additive Manufacturing, 2016. 12: p. 240-251.

[15] Nickel, A., D. Barnett, and F. Prinz, Thermal stresses and deposition patterns in layered manufacturing. Materials Science and Engineering: A, 2001. 317(1-2): p. 59-64.
[16] Töppel, T., et al., Eigenspannungen und Verzug beim additiven Laser-Strahlschmelzen. Schweißen und Schneiden, 2016. 68(4): p. 176-186.

[17] Zaeh, M.F. and G. Branner, Investigations on residual stresses and deformations in selective laser melting. Production Engineering, 2010. 4(1): p. 35-45.

[18] Kruth, J.-P., et al. Part and material properties in selective laser melting of metals. in Proceedings of the 16th international symposium on electromachining. 2010.

[19] Carter, L.N., et al., The influence of the laser scan strategy on grain structure and cracking behaviour in SLM powder-bed fabricated nickel superalloy. Journal of Alloys and Compounds, 2014. 615: p. 338-347.

[20] Li, C., et al., A multiscale modeling approach for fast prediction of part distortion in selective laser melting. Journal of materials processing technology, 2016. 229: p. 703-712.

[21] Kruth, J.-P., et al., Selective laser melting of iron-based powder. Journal of materials processing technology, 2004. 149(1-3): p. 616-622.

[22] Ali, H., H. Ghadbeigi, and K. Mumtaz, Effect of scanning strategies on residual stress and mechanical properties of Selective Laser Melted Ti6Al4V. Materials Science and Engineering: A, 2018. 712: p. 175-187.

[23] Hagedorn-Hansen, D., et al., The effects of selective laser melting scan strategies on deviation of hybrid parts. South African Journal of Industrial Engineering, 2017. 28(3): p. 200212.

[24] Valente, E.H., et al., Effect of Scanning Strategy During Selective Laser Melting on Surface Topography, Porosity, and Microstructure of Additively Manufactured Ti-6Al-4V. Applied Sciences, 2019. 9(24): p. 5554.

[25] Lu, Y., et al., Study on the microstructure, mechanical property and residual stress of SLM Inconel-718 alloy manufactured by differing island scanning strategy. Optics \& Laser Technology, 2015. 75: p. 197-206.

[26] Malekipour, E., S. Attoye, and H. El-Mounayri, Investigation of layer based thermal behavior in fused deposition modeling process by infrared thermography. Procedia Manufacturing, 2018. 26: p. 1014-1022.

[27] Qiu, C., et al., A comprehensive study on microstructure and tensile behaviour of a selectively laser melted stainless steel. Scientific reports, 2018. 8(1): p. 1-16.

[28] Yeung, H., et al. Continuous laser scan strategy for faster build speeds in laser powder bed fusion system. in Proceedings of the 28th Annual International Solid Freeform Fabrication Symposium. 2017.

[29] Wiesner, A. and D. Schwarze. Multi-laser selective laser melting. in 8th International Conference on Photonic Technologies LANE. 2014.

[30] Masoomi, M., S.M. Thompson, and N. Shamsaei, Quality part production via multi-laser additive manufacturing. Manufacturing letters, 2017. 13: p. 15-20.

[31] Zitelli, C., P. Folgarait, and A. Di Schino, Laser powder bed fusion of stainless steel grades: a review. Metals, 2019. 9(7): p. 731. 
[32] Malekipour, E., Y. Shin, and H. El-Mounayri, Optimization of Chessboard Scanning Strategy Using Genetic Algorithm in Multi-laser Additive Manufacturing Process. 2020.

[33] Malekipour, E., Y. Shin, and H. El-Mounayri, Optimization of Chessboard Scanning Strategy by Using Genetic Algorithm in Powder-bed Fusion Process. 2020.

[34] Malekipour, E., et al., A Vision toward Layer-wise Intelligent Monitoring and Control of Scan Strategy in Powderbed Fusion Process. TechConnect Briefs, 2019. TechConnect Briefs 2019: p. 127 - 130. 